\title{
CONSUMO DE SUPLEMENTOS NUTRICIONALES EN GIMNASIOS, PERFIL DEL CONSUMIDOR Y CARACTERÍSTICAS DE SU USO
}

\section{NUTRITIONAL SUPPLEMENT INTAKE IN GYMNASIUM, CONSUMER PROFILE AND CHARATERISTICS OF THEIR USE}

\author{
Fernando Rodríguez R. (1), Mirta Crovetto M. (2) Andrea González A. (2), \\ Nikol Morant C. (2), Francisco Santibáñez T. (2) \\ (1) Escuela de Educación Física, Facultad de Filosofía y Educación, \\ Pontificia Universidad Católica de Valparaíso, Valparaíso, Chile. \\ (2) Nutrición y Dietética, Facultad de Ciencias de la Salud, \\ Universidad de Playa Ancha, Valparaíso, Chile.
}

\begin{abstract}
Nowadays the great offer of dietary supplements (DS) in the market has led to a situation in which the use of these products are not only acquired by sportspersons but also to a great population related with physical activity. The main objective of our study was to know the characteristics of the DS consumers and to evaluate its consumption through a survey previously validated and applied to 314 users (198 males and 116 females) from 6 gyms in Viña del Mar. The results showed that more than half of the subjects consumed DS products $(54.5 \%)$. Within male consumers, a $69.4 \%$ use these products in order to increase muscular mass, while within women, a $62.2 \%$ consumed them in order to diminish their body fat. There were 5 kinds of DS products consumed the most: Proteins (55.6\%), amino acids (25.9\%), vitamins and minerals (25.1\%), food substitutes (6.4\%), caffeine (6.4\%), and L-carnitine (6.4\%). The consumer profile is represented by young male individuals, those who follow a special diet, with long practice in gym and who exercise several days a week. Much of consumers did not provide an adequate association between DSN used and the purpose of consuming these supplements.
\end{abstract}

KEY WORDS: Nutritional supplement; exercise; sport; sport nutrition; performance.

Este trabajo fue recibido el 5 de Octubre de 2010 y aceptado para ser publicado el 7 de Abril de 2011.

\section{INTRODUCCIÓN}

En el mundo actual, al analizar la alimentación de la población, ya no basta considerar únicamente los alimentos, si no que se debe tener presente otras alternativas cuando los requerimientos de nutrientes son elevados, tales como los suplementos nutricionales ( $\mathrm{SN}$ ), los cuales muchas veces son utilizados como complemento a la dieta, sin mayor respaldo científico que justifique su uso $(1,2)$. Según el Reglamento Sanitario de los Alimentos, un $\mathrm{SN}$, es un producto que ha sido elaborado para suplementar la dieta habitual de los individuos sanos, con adición de uno o varios nutrientes y que se presenta en diversos formatos. Estos productos, suelen estar acompañados de una propaganda comercial que los presentan como sustancias milagrosas que contribuyen a diversos objetivos para mantener la salud.
El crecimiento de la industria de gimnasios en Chile, ha provocado un aumento en el número de personas que acuden regularmente a estos centros. La inserción a este medio, predispone al individuo a consumir SN por asesorías de profesionales, que muchas veces, no se encuentran debidamente capacitados para ejecutar esta labor (3 - 5). Son pocos los estudios científicos que dan cuenta del consumo de SN por la población cercana al mundo de la actividad física (5). Nuestro país, no difiere de esta realidad al no presentar estudios al respecto. $\mathrm{La}$ escasez de información existente, dificulta la orientaciones necesarias para abordar la problemática del uso, mal uso y abuso de los SN, por lo que el objetivo general de esta investigación, es conocer las características que determinan el consumo de $\mathrm{SN}$ en gimnasios de la comuna de Viña del Mar, evaluando su consumo mediante la 
TABLA 1

Total de sujetos de la muestra que consumen suplementos nutricionales

¿Consume algún tipo de SN?

\begin{tabular}{cccccc}
\hline & \multicolumn{2}{c}{ Sí } & \multicolumn{2}{c}{ No } & \multicolumn{2}{c}{ Total } \\
\hline $\mathrm{n}$ & $\%$ & $\mathrm{n}$ & $\%$ & $\mathrm{n}$ & $\%$ \\
171 & $54,5 \%$ & 143 & $45,5 \%$ & 314 & $100,0 \%$
\end{tabular}

\section{TABLA 2}

Caracterización de la muestra según años, días y horas de práctica de actividad física y tipo de dieta

n $\quad \%$

\begin{tabular}{|c|c|c|c|}
\hline ¿Cuánto tiempo & Menos de un ańo & 111 & 35,4 \\
\hline lleva practic ando en & Un año & 126 & 40,1 \\
\hline \multirow[t]{2}{*}{ el gimnasio? } & Más de un año & 77 & 24,5 \\
\hline & Total & 314 & 100,0 \\
\hline \multirow{8}{*}{$\begin{array}{l}\text { ¿Cuántos dias } \\
\text { asiste al gimnasio? }\end{array}$} & Dos veces por & 34 & 10.8 \\
\hline & semana & & \\
\hline & Tres veces por & 132 & 42,0 \\
\hline & semana & & \\
\hline & Cinco veces por & 105 & 33,4 \\
\hline & semana & & \\
\hline & Todos los dias & 43 & 13.7 \\
\hline & Total & 314 & 100,0 \\
\hline ¿Cuántas horas & Una hora & 36 & 11,5 \\
\hline entrena en el & Tres Horas & 147 & 46,8 \\
\hline \multirow[t]{2}{*}{ gimnasio? } & Más de tres horas & 131 & 41,7 \\
\hline & Total & 314 & 100,0 \\
\hline ¿Sigue algún tipo de & Si & 169 & 53,8 \\
\hline dieta, o & No & 145 & 46,2 \\
\hline alimentación & Total & 314 & 100.0 \\
\hline especial? & & & \\
\hline
\end{tabular}


aplicación de una encuesta, que determinó las principales conductas y perfil de la población consumidora.

\section{SUJETOS Y MÉTODO}

El universo está compuesto por el número de inscritos en gimnasios de la ciudad de Viña del Mar, registrados en el último mes previo a la aplicación del instrumento de medición de la investigación. Este universo presenta una rotación constante de usuarios, determinada por los diversos horarios, irregularidad en la asistencia y número de inscripciones por estacionalidad. Todas estas características hacen que este universo sea de difícil acceso para el llenado del instrumento, invalidando la opción de una muestra con características de aleatoridad, por lo cual se seleccionó una muestra intencionada con el objetivo de abarcar el mayor número posible de sujetos. Los sujetos fueron voluntarios, quienes accedieron a participar firmando un consentimiento informado. Se aplicó un cuestionario previamente validado en una muestra de 120 estudiantes universitarios. El instrumento es estructurado en base a preguntas cerradas, con alternativas y preguntas abiertas, para recabar información acerca del consumo de $\mathrm{SN}$, objetivos de consumo, frecuencia, lugar de procedencia y relación entre consumo y objetivos a alcanzar. La validación del instrumento de medición fue realizada en dos etapas, en una primera instancia se validó en un grupo de deportistas a través de formato electrónico, realizándose las modificaciones pertinentes. Posteriormente, se volvió a aplicar en usuarios regulares de cinco gimnasios de la comuna de Viña del Mar, distintos de los gimnasios correspondientes a la muestra. Luego de este proceso, se realizaron las últimas modificaciones en la estructura del cuestionario obteniendo el instrumento definitivo.

La población objetivo fueron 314 usuarios hombres y mujeres de los gimnasios seleccionados con un rango

TABLA 3

Consumo de suplementos nutricionales según sexo

\begin{tabular}{rrrrrrr}
\hline \multicolumn{7}{c}{ Sexo del entrevistado } \\
\hline & \multicolumn{1}{c}{ Hombre } & \multicolumn{1}{c}{ Mujer } & \multicolumn{2}{c}{ Total } \\
\hline Proteínas & 87 & 64,9 & 8 & 21,6 & 95 & 55,6 \\
Aminoácidos & 42 & 31,6 & 2 & 5,4 & 44 & 25,9 \\
Vit y min & 23 & 17,2 & 20 & 54,1 & 43 & 25,1 \\
Creatina & 13 & 9,7 & 4 & 10,8 & 17 & 9,9 \\
Sust de comida & 8 & 6,0 & 3 & 8,1 & 11 & 6,4 \\
Cafeína & 7 & 5,2 & 4 & 10,8 & 11 & 6,4 \\
L- carnitina & 7 & 5,2 & 4 & 10,8 & 11 & 6,4 \\
Glutamina & 5 & 3,7 & 0 & 0 & 5 & 2,9 \\
Carbohidratos & 4 & 3,0 & 0 & 0 & 4 & 2,3 \\
Ginseng & 3 & 2,2 & 1 & 2,7 & 4 & 2,3 \\
Arginina & 3 & 2,2 & 0 & 0 & 3 & 1,8 \\
Guaraná & 1 & 0,7 & 0 & 0 & 1 & 0,6 \\
HCA & 1 & 0,7 & 0 & 0 & 1 & 0,6
\end{tabular}


de edad entre 15 a 65 años. La encuesta se aplicó en un período de dos semanas, en la jornada de mañana y tarde y en los horarios de asistencia de los encuestados a los gimnasios definidos, a través de encuestadores previamente capacitados. Los datos fueron analizados utilizando el software estadístico SPSS Estadistics, $17^{\mathrm{a}}$ edición, considerando un nivel de significancia de $\mathrm{p}<0,00$ para la comparación de las variables, en la prueba Chi cuadrado.

\section{RESULTADOS}

De los 314 encuestados un 63,1\% pertenece al sexo masculino, mientras que un $36,9 \%$ son de sexo femenino. La mayoría de los encuestados tenía un rango de edad entre 15 a 30 años (51,9\%), seguido por el rango de edad que varía entre los 31 a los 45 años $(38,9 \%)$ y un grupo menor que pertenece al rango de 46 años o más $(9,2 \%)$.

En cuanto al tipo de ocupación de los encuestados, la mayoría señala ser estudiante $(49,4 \%)$, seguido por tra- bajadores $(46,2 \%)$ y sólo un pequeño porcentaje $(2,9 \%)$ declaró estar inactivo o jubilado (1,6\%). Un 54,5\% del total de encuestados señala consumir algún tipo de $\mathrm{SN}$, mientras el 45,5\% declaró no consumirlos (tabla 1).

La tabla 2 indica los años, días y horas de práctica, y el seguimiento de algún tipo de dieta especial según sexo de la muestra.

Los SN más consumidos son proteínas $(55,6 \%)$, aminoácidos $(25,9 \%)$ y vitaminas y minerales $(25,1 \%)$. Los SN menos consumidos son arginina $(1,8 \%)$, guaraná $(0,6 \%)$ y $\mathrm{HCA}(0,6 \%)$ (tabla 3$)$.

Los datos presentados en la tabla 4 nos permiten señalar que aproximadamente siete de cada diez hombres usuarios de la muestra de gimnasios de Viña del Mar consumen algún tipo de SN. Tres de cada diez mujeres usuarias de gimnasios de Viña del Mar consumen algún tipo de SN. El número de hombres que consumen SN es mayor al número de mujeres.

La tabla 5 muestra que $87 \%$ de los usuarios de gim-

TABLA 4

Consumo de suplementos nutricionales según sexo del usuario

\begin{tabular}{|c|c|c|c|c|c|c|}
\hline \multirow{3}{*}{$\begin{array}{r}\text { Sexo del } \\
\text { entrevistado }\end{array}$} & \multicolumn{6}{|c|}{ ¿Consume algún tipo de suplemento nutricional? } \\
\hline & \multicolumn{2}{|c|}{ Sí } & \multicolumn{2}{|c|}{ No } & \multicolumn{2}{|c|}{ Total } \\
\hline & $n$ & $\%$ & $\mathrm{n}$ & $\%$ & $\mathrm{n}$ & $\%$ \\
\hline Masculino & 134 & 67.7 & 64 & 32,3 & 198 & 100,0 \\
\hline Femenino & 37 & 31,9 & 79 & 68.1 & 116 & 100,0 \\
\hline Total & 171 & 54.5 & 143 & 45,5 & 314 & 100,0 \\
\hline
\end{tabular}

TABLA 5

Consumo de suplementos nutricionales según edad del usuario

\begin{tabular}{|c|c|c|c|c|c|c|}
\hline \multirow{3}{*}{ Rango de edad } & \multicolumn{6}{|c|}{ ¿Consume algún tipo de suplemento nutricional? } \\
\hline & \multicolumn{2}{|c|}{$\mathrm{Si}$} & \multicolumn{2}{|c|}{ No } & \multicolumn{2}{|c|}{ Total } \\
\hline & $\mathrm{n}$ & $\%$ & $\mathrm{n}$ & $\%$ & $\mathrm{n}$ & $\%$ \\
\hline De 15 a 30 años & 142 & 87.1 & 21 & 12,9 & 163 & 100,0 \\
\hline De 31 a 45 años & 24 & 19.7 & 98 & 80,3 & 122 & 100,0 \\
\hline 46 años o más & 5 & 17,2 & 24 & 82,8 & 29 & 100,0 \\
\hline
\end{tabular}


nasios pertenecen al rango de edad que va desde los 15 a los 30 años consumen SN. Es decir, aproximadamente nueve de cada diez usuarios encuestados de gimnasios de entre 15 y 30 años consumen SN. Aproximadamente, dos de cada cinco encuestados de gimnasios que pertenecen al rango de edad que entre los 31 a 45 años consumen algún tipo de SN. Sólo un 17,2\% de los usuarios de gimnasios que tienen más de 45 años consumen algún tipo de SN.

La prueba de Chi Cuadrado entrega una asociación muy significativa entre las dos variables $(p<0,00)$. Es decir, el consumo de SN se encuentra asociado al rango de edad de pertenencia de los mismos, siendo mayor el consumo entre los jóvenes de 15 a 30 años y disminuyendo a medida que aumenta el rango de edad.

Los datos de la tabla 6, nos permiten establecer que ocho de cada diez usuarios de gimnasios que declaran estar estudiando, consume algún tipo de SN. Sólo un $27,6 \%$ de los usuarios de gimnasios que declara estar trabajando consume algún tipo de SN. Un tercio $(33,3 \%)$ de los usuarios de gimnasios que se declaran inactivos consumen algún tipo de SN. Sólo un $20 \%$ de los usuarios de gimnasios que se declararon pensionados consume algún tipo de SN.

La aplicación del estadístico Chi Cuadrado entrega una asociación muy significativa entre las dos variables $(\mathrm{p}<0,00)$. Es decir que el consumo de SN está muy asociado al tipo de ocupación de los usuarios de gimnasios.

Objetivos del consumo de SN. La tabla 7 muestra que los hombres tienen como principal objetivo el desarrollo muscular $(69,4 \%)$, seguido por rendimiento deportivo (17,9\%), obtener energía $(6,7 \%)$ y finalmente disminuir grasa corporal y salud (3\%). Respecto a las mujeres, la mayoría utiliza SN con el objetivo de dis-

\section{TABLA 6}

Consumo de suplementos nutricionales según tipo de educación del usuario

\begin{tabular}{|c|c|c|c|c|c|c|}
\hline \multirow[b]{3}{*}{ Tipo ocupación } & \multicolumn{6}{|c|}{ ¿Consume algún tipo de suplemento nutricional? } \\
\hline & \multicolumn{2}{|c|}{ Sí } & \multicolumn{2}{|c|}{ No } & \multicolumn{2}{|c|}{ Total } \\
\hline & $\mathrm{n}$ & $\%$ & $\mathrm{n}$ & $\%$ & $\mathrm{n}$ & $\%$ \\
\hline Estudiando & 127 & 81,9 & 28 & 18,1 & 155 & 100,0 \\
\hline Trabajando & 40 & 27,6 & 105 & 72,4 & 145 & 100,0 \\
\hline Inactivo & 3 & 33,3 & 6 & 66,7 & 9 & 100,0 \\
\hline Jubilado / pensionado & 1 & 20,0 & 4 & 80,0 & 5 & 100,0 \\
\hline Dueño (a) de casa & 0 & 0 & 0 & 0 & 0 & 0 \\
\hline
\end{tabular}

TABLA 7

Clasificación del objetivo por el cual consume suplementos nutricionales, según sexo

\begin{tabular}{|c|c|c|c|c|c|c|c|c|c|c|c|c|}
\hline \multirow{3}{*}{$\begin{array}{l}\text { Sexo del } \\
\text { entrevistado }\end{array}$} & \multicolumn{12}{|c|}{ ¿Con qué objetivo lo consume? } \\
\hline & \multicolumn{2}{|c|}{$\begin{array}{l}\text { Rendimiento } \\
\text { deportivo }\end{array}$} & \multicolumn{2}{|c|}{$\begin{array}{l}\text { Desarrollo } \\
\text { muscular }\end{array}$} & \multicolumn{2}{|c|}{$\begin{array}{l}\text { Obtener } \\
\text { energía }\end{array}$} & \multicolumn{2}{|c|}{$\begin{array}{c}\text { Disminuir } \\
\text { grasa } \\
\text { corporal }\end{array}$} & \multicolumn{2}{|c|}{ Salud } & \multicolumn{2}{|c|}{ Total } \\
\hline & $\mathrm{n}$ & $\%$ & $\mathrm{n}$ & $\%$ & $\mathrm{n}$ & $\%$ & $\mathrm{n}$ & $\%$ & $\mathrm{n}$ & $\%$ & $\mathrm{n}$ & $\%$ \\
\hline Masculino & 24 & 17,9 & 93 & 69,4 & 9 & 6,7 & 4 & 3,0 & 4 & 3,0 & 134 & 100,0 \\
\hline Femenino & 2 & 5,4 & 3 & 8,1 & 4 & 10,8 & 23 & 62,2 & 5 & 13,5 & 37 & 100,0 \\
\hline Total & 26 & 15,2 & 96 & 56,1 & 13 & 7,6 & 27 & 15,8 & 9 & 5,3 & 171 & 100,0 \\
\hline
\end{tabular}


minuir grasa corporal $(62,2 \%)$. Otro de los objetivos de consumo fueron: salud $(13,5 \%)$, obtener energía $(10,8 \%)$, desarrollo muscular $(8,1 \%)$ y la minoría por rendimiento deportivo $(5,4 \%)$.

Asesoría profesional para el consumo SN. Los resultados de la tabla 8, en relación a la asesoría del consumo de $\mathrm{SN}$ indican que los hombres fueron asesorados en su mayoría por un entrenador $(48,5 \%)$, amigo $(17,2 \%)$, nutricionista $(14,2 \%)$ ó médico $(9 \%)$. Una minoría declara haber sido incentivado por la publicidad $(4,5 \%)$, por el farmacéutico $(2,2 \%)$ y el resto a través de "otro" tipo de asesoría (4,5\%). En mujeres la asesoría estuvo dada por algún entrenador $(37,8 \%)$, amigo (27\%), nutricionista $(13,5 \%)$ y médico $(8,1 \%)$. Algunas fueron motivadas por "otro" tipo de asesoría $(8,1 \%)$ y en menor proporción a través de la publicidad $(5,4 \%)$.

La mayor cantidad de respuestas tanto en hombres como mujeres indicaron que la asesoría fue a través de un entrenador $(46,2 \%)$.

Efectos esperados. Respecto al logro de los efectos esperados a través del consumo del SN (Tabla 9), la mayoría de los encuestados $(84,8 \%)$ respondieron si haber logrado los efectos deseados, tanto hombres $(85,8 \%)$ como mujeres $(81,1 \%)$. Los individuos que declararon no haber logrado los efectos esperados representan la minoría de los encuestados $(15,2 \%)$.

\section{DISCUSIÓN}

El consumo de SN responde a distintas motivaciones, los de mayor consumo se encuentran los suplementos en a base a proteínas y aminoácidos cuyos efectos anabólicos han sido científicamente comprobados (6 -10). El $64,2 \%$ de los consumidores de proteínas, y $65,9 \%$ de aminoácidos, los consumían con este objetivo. Del total de usuarios consumidores de SN, $25,1 \%$ consumía complejos de vitaminas y/o minerales. La mayoría los consume con el objetivo de desarrollar masa muscular $(32,6 \%)$ y disminuir grasa corporal $(30,2 \%)$. Tan sólo una minoría lo consumía por salud $(9,3 \%)$ y para obtener energía $(9,3 \%)$. A este tipo de $\mathrm{SN}$ se les ha atribuido un efecto protector de la salud, dado su poder antioxidante, especialmente por la vitamina E, vitamina $\mathrm{C}$ y selenio. Otras vitaminas de este complejo, participan en la producción de eritrocitos y síntesis proteica, así como también en la reparación y mantenimiento de los tejidos, por lo que se podría fundamentar su consumo con el objetivo de desarrollar masa muscular. Aún así, existe controversia en la capacidad ergogénica de este tipo de suplementos, debido a que para la mayoría de las vitaminas y minerales no se ha comprobado este mecanismo de acción $(11,12)$.

Del total de consumidores de $\mathrm{SN}$ como sustitutivos de comidas $(6,4 \%)$, el mayor porcentaje lo consumía para

\section{TABLA 8}

Distribución del tipo de asesoría que incentivó el consumo de suplementos nutricionales según sexo

\section{Sexo del entrevistado}

\begin{tabular}{rrrrrrr} 
¿Quién le recomendó & \multicolumn{2}{c}{ Masculino } & \multicolumn{2}{c}{ Femenino } & \multicolumn{2}{c}{ Total } \\
\cline { 2 - 8 } el suplemento? & \multicolumn{1}{c}{$\mathrm{n}$} & $\%$ & $\mathrm{~N}$ & $\%$ & $\mathrm{n}$ & $\%$ \\
Médico & 12 & 9,0 & 3 & 8,1 & 15 & 8,8 \\
Nutricionista & 19 & 14,2 & 5 & 13,5 & 24 & 14,0 \\
Farmacéutico & 3 & 2,2 & 0 & 0 & 3 & 1,8 \\
Entrenador & 65 & 48,5 & 14 & 37,8 & 79 & 46,2 \\
Publicidad & 6 & 4,5 & 2 & 5,4 & 8 & 4,7 \\
Amigo & 23 & 17,2 & 10 & 27,0 & 33 & 19,3 \\
Otro & 6 & 4,5 & 3 & 8,1 & 9 & 5,3 \\
Total & 134 & 100,0 & 37 & 100,0 & 171 & 100,0
\end{tabular}


desarrollo muscular (54,5\%), mientras que en menor porcentaje con el objetivo de proveer mayor rendimiento deportivo (18,2\%), disminuir grasa corporal $(18,2 \%)$, y por motivos de salud $(9,1 \%)$. Sin embargo, estos productos son elaborados con la finalidad de sustituir uno o más tiempos de comida al día, proporcionando una dieta de baja densidad calórica $(13,14)$. En efecto, el uso de este tipo de SN en conjunto con una dieta baja en calorías, ha sido considerado como un método factible para el control del peso (13 - 18). Aún así, sólo un pequeño porcentaje los consumía como Sustitutivos de comida para disminuir el peso.

Respecto al total de individuos que consumía cafeína $(6,4 \%)$, la mayoría tenía como objetivo aumentar su masa muscular $(72,7 \%)$, mientras que el otro objetivo era disminuir grasa corporal $(18,2 \%)$ y obtener energía $(9,1 \%)$. El primer objetivo con mayor prevalencia, no posee respaldo científico, por lo cual se estaría haciendo mal uso de este suplemento. No así, las siguientes alternativas, en donde la documentación señala que la Cafeína contribuye a la pérdida de grasa corporal, al aumentar el gasto energético basal y la disponibilidad de ácidos grasos libres para favorecer su utilización como sustrato energético (19-22). Esto, contribuye al ahorro de glucógeno, al aumento de la contracción muscular y resistencia al ejercicio, efectos que le permiten ser considerada como una ayuda ergogénica (23-26).

Del total de consumidores de L-carnitina $(6,4 \%)$, la mayoría la consumía para aumentar masa muscular $(54,5)$ y para disminuir grasa corporal $(36,4 \%)$. La Lcarnitina es conocida como una sustancia lipotrópica. Sin embargo, existen múltiples estudios que no han podido demostrar la veracidad de esta teoría, refutando que la L-carnitina acelere los procesos lipolíticos (27 - 30), por

\section{TABLA 9}

Distribución de los tipos de suplementos nutricionales y objetivos de su consumo

¿Con qué objetivo lo consume?

\begin{tabular}{lrrrrr} 
& $\begin{array}{c}\text { Rendimiento } \\
\text { deportivo }\end{array}$ & $\begin{array}{c}\text { Desarrollo } \\
\text { muscular }\end{array}$ & $\begin{array}{c}\text { Obtener } \\
\text { energia }\end{array}$ & $\begin{array}{c}\text { Disminuir } \\
\text { grasa } \\
\text { corporal }\end{array}$ & Salud \\
\hline Proteinas & \multicolumn{1}{c}{$\%$} & \multicolumn{1}{c}{$\%$} & \multicolumn{1}{c}{$\%$} & $\%$ \\
Aminoácidos & 14,7 & 64,2 & 7,4 & 11,6 & 2,1 \\
Vit y min & 13,6 & 65,9 & 11,3 & 2,3 & 6,8 \\
Creatina & 18,6 & 32,6 & 9,3 & 30,2 & 9,3 \\
Sust de comida & 23,5 & 52,9 & 5,9 & 17,6 & 0 \\
Cafeina & 18,2 & 54,5 & 0 & 18,2 & 9,1 \\
L-camitina & 0 & 72,7 & 9,1 & 18,2 & 0 \\
Glutamina & 9,1 & 54, & 0 & 36,4 & 0 \\
Carbohidratos & 40,0 & 40,0 & 20,0 & 0 & 0 \\
Ginseng & 50,0 & 25,0 & 25,0 & 0 & 0 \\
Arginina & 0 & 75,0 & 0 & 25,0 & 0 \\
Guarana & 0 & 66,7 & 0 & 33,3 & 0 \\
HCA & $0 \%$ & 100 & 0 & 0 & 0 \\
Picolinato de & $0 \%$ & 100 & 0 & 0 & 0 \\
Cromo & $0 \%$ & 0 & 0 & 0 & 0 \\
CLA & $0 \%$ & 0 & 0 & 0 & 0
\end{tabular}


lo cual no estaría justificado su uso para ninguna de las opciones mencionadas anteriormente.

A pesar de lo anteriormente mencionado, al preguntarles a los consumidores si lograron los efectos esperados a través del consumo de SN, el 84,8\% del total, refiere haber logrado los efectos esperados, aún cuando consumen $\mathrm{SN}$ cuyos efectos no han sido comprobados científicamente. Por lo tanto, en este aspecto, podemos atribuir que estos logros posiblemente son el resultado del efecto placebo ó de algún otro factor no evaluado a través del cuestionario aplicado.

Si bien dentro los SN más consumidos, se encuentra una cantidad importante de individuos que los utiliza adecuadamente en relación al objetivo, existe un porcentaje no menor de sujetos que presentan incongruencias entre el consumo y el objetivo a conseguir a través de su consumo.

De acuerdo a la evidencia científica, existen numerosos SN que contribuirían al logro de los objetivos perseguidos por los consumidores (31 - 38), sin embargo, por falta de conocimientos o por ausencia de una asesoría profesional, los $\mathrm{SN}$ consumidos no son los más adecuados en relación a los objetivos que el consumidor quiere obtener.

Este estudio determinó que de la totalidad de los asistentes a gimnasios encuestados $(\mathrm{n}=314)$, más de la mitad consumía, algún tipo de $\mathrm{SN}$, en el momento de aplicación de la encuesta siendo los más consumidos: Proteínas, aminoácidos, vitaminas y minerales, sustitutivos de comida, cafeína y L- carnitina.

Los hombres presentaron un mayor consumo de SN que las mujeres, estas últimas además, presentaron una menor frecuencia y cantidad de SN utilizados.

Las motivaciones del consumo de SN también diferían por sexo. Las mujeres buscaban en su mayoría disminuir grasa corporal, mientras que los hombres buscaban un mayor desarrollo muscular. Esto, diferencia el SN elegido por cada uno de ellos, ya que los hombres consumían más Proteínas y las mujeres vitaminas y minerales.

$\mathrm{Al}$ evaluar la relación entre los $\mathrm{SN}$ de mayor consumo encontrados en este estudio con el objetivo que motivaba su consumo, encontramos una amplia cantidad de individuos que realizaban una inadecuada asociación. Esto se atribuye en gran parte a la asesoría técnica que presenta el grupo de estudio, que en su mayoría recibían orientaciones de entrenadores o amigos.

Esta investigación no presenta una muestra significativa que nos permita extrapolar los resultados al resto de la población, aun así, la información obtenida constituye una línea base para los profesionales en el área, especialmente el profesional nutricionista, quienes deben asesorar adecuadamente a los individuos consumidores a través de fundamentos científicos sólidos que resguarden una ingesta segura y que sea suficientemente efectiva para alcanzar los objetivos perseguidos.

Se requieren de más estudios al respecto, ya que el número de individuos pesquisados en esta investigación que consume $\mathrm{SN}$ no es menor. Además, si consideramos que actualmente estos productos están a disposición de cualquier individuo, es de vital importancia profundizar en el tema para determinar si es necesaria una legislación que controle la libre oferta y demanda de estos productos.

\section{RESUMEN}

La gran oferta de suplementos nutricionales (SN) dispuestos en el mercado, ha llevado a que el uso de estos productos no sea adquirido únicamente por deportistas, sino que por parte importante de la población relacionada con la actividad física. El objetivo de este estudio es conocer las características de los consumidores de $\mathrm{SN}$, evaluando su consumo en 314 usuarios (198 hombres y 116 mujeres) de 6 gimnasios de la ciudad de Viña del Mar. Los resultados revelan que más de la mitad de los usuarios consumen SN (54,5\%). De los hombres consumidores, un $69,4 \%$ lo realiza para aumentar masa muscular, mientras que del total de las mujeres, un $62,2 \%$ lo utiliza para disminuir grasa corporal. Los cinco tipos de SN más consumidos en relación a su composición de nutrientes y otras fuentes fueron: proteínas $(55,6 \%)$, aminoácidos $(25,9 \%)$, vitaminas y minerales $(25,1 \%)$, sustitutivos de comida $(6,4 \%)$, cafeína $(6,4 \%)$ y L- carnitina $(6,4 \%)$. El perfil del consumidor, está representado por individuos jóvenes, de sexo masculino, los que siguen una dieta especial de alimentación, con largo tiempo de práctica en gimnasio y que se ejercitan varios días y horas a la semana. Gran parte de los consumidores no presentó una adecuada asociación entre el SN utilizado y el objetivo de consumo de estos suplementos.

Palabras clave: suplementos nutricionales; ejercicio; deporte; nutrición deportiva; rendimiento físico.

Dirigir la correspondencia a:

Profesora

Mirta Crovetto Mattassi

Nutrición y Dietética

Facultad de Ciencias de la Salud

Universidad de Playa Ancha

Valparaíso,

Chile

Fono: (32) 2500100

E-mail:mirtacrovetto@gmail.com 


\section{BIBLIOGRAFÍA}

1. Haymes, E.M. Vitamin and mineral supplementation to athletes. Int J Sports Nutr 1991, 1(2): 146-69.

2. Petróczi, A., Naughton, DP., Pearce, G., Bailey, R., Bloodworth, A., McNamee, M. Nutritional supplement use by elite young UK athletes: fallacies of advice regarding efficacy. J Int Soc Sports Nutr 2008, 5: 22.

3. Alves dos Santos, M.A., \& Pereira dos Santos, R. Uso de suplementos alimentares como forma de melhorar a performance nos programas de atividade física em academias de ginástica. Rev Paul Educ Fís. 2002. 16(2):174-85.

4. Maughan, R.J., King, D.S., \& Lea, T. Dietary supplements. J Sports Sci 2004, 22: 95-113.

5. Sánchez, A., Miranda, M.T., \& Guerra, E. "Estudio estadístico del consumo de suplementos nutricionales y dietéticos en gimnasios". Arch Latinoam Nutr 2008, 58(3): 221-27.

6. Biolo, G., Tipton, K., Klein, S., \& Wolfe, R. An abundant supply of amino acids enhances the metabolic effect of exercise on muscle protein. Am J Physiol 1997, 273: 122-29.

7. Tipton, K., Gurkin, B., Matin, S., \& Wolfe, R. Nonessential amino acids are not necessary to stimulate net muscle protein synthesis in healthy volunteers. J Nutr Biochem 1999, 10(2): 89-95.

8. Rasmussen, B., Tipton, K., Miller, S., Wolf, S., \& Wolf, R. An oral essential amino acid-carbohydrate supplement enhances muscle protein anabolism after resistance exercise. J Appl Physiol 2000, 88:386-392.

9. Andersen, L., Tufekovic, G., Zebis, M., Crameri, R., Verlaan, G., Kjaer, M., Suetta, C., Magnusson, P., \& Aagaard, P. The effect of resistance training combined with timed ingestion of protein on muscle fiber size and muscle strength. Metabolism 2005, 54(2): 151-6.

10. Cribb, P., \& Hayes, A.. Effects of supplement timing and resistance exercise on skeletal muscle hypertrophy. Med Sci Sports Exerc 2006, 38(11): 1918-25.

11. Clarkson, P., \& Thompson, H. Antioxidants: "What role do they play in physical activity and health". Am J Clin Nutr 2000, 72(2): 637-46.

12. Grandjean AC. Vitamine, diet and the athlete. Clin Sports Med 1983, 2:105-14.

13. Noakes, M., Keogh, J. \& Foster, P. How effective are meal replacements for treating obesity? J Nutr 2004, 134: 1894-9.

14. Cabanillas, M., Moya, E., González, C., Loria, V., Dassen, C \& Lajo, T. (2009). Características y utilidad de los sustitutos de la comida: análisis de los productos comercializados de uso habitual en nuestro entorno. Nutr Hospitalaria 2009; 24(5):535-42.

15. Ashley, J., Sachiko, T., Perumean-Chaney, S., Schrage, J., \& Bovee, V. Meal replacements in weight intervention. Obes Res 2001, 9: 312-20.

16. Ashley, J., Sachiko T., Schrage, J., PerumeanChaney, S., Gilbertson,M., McCall N.,\& Bovee,V. Weight Control in the Physician's Office. Arch Intern Med 2001, 161: 1599-616.

17. Vidal-Guevara, M., Samper, M., Martínez-Silla, G., Canteras, C., Ros, G., Gil, A \& Abellán, P. Sustitutivos de una comida como terapia dietética en el control de peso. Evaluación en hombres y mujeres con diferentes grados de obesidad. Nutr Hosp 2004, 19(4): 202-208.

18. Treyzon, L., Chen, S., Hong, K., Yan, E., Carpenter, C., Thames, G., Bowerman, S., Wang, H., Elashoff, R., \& Li, Z. A controlled trial of protein enrichment of meal replacements for weight reduction with retention of lean body mass. J Nutr 2008, 7:23.

19. Dodd SL, Herb RA, and Powers SK. Caffeine and exercise performance. An update. Sports Med 1993, 15: 14-23.

20. Graham TE. Caffeine and exercise: metabolism, endurance and performance. Sports Med 2001, 31: 785-807.

21. Pasman WJ, van Baak MA, Jeukendrup AE, and de Haan A. The effect of different dosages of caffeine on endurance performance time. Int J Sports Med 1995, 16: 225-30.

22. Graham TE and Spriet LL. Metabolic, catecholamine, and exercise performance responses to various doses of caffeine. J Appl Physiol 1995, 78: 867-74.

23. Cox G, Desbrow B, Montgomery P, Anderson M, Bruce C, Macrides T, Martin D, Moquin A, Roberts A, Hawley J, Burke L, Effect of different protocols of caffeine intake on metabolism and endurance performance. J Appl Physiol 2002. 93; 990_-9

24. Innkins N, Trilk J. Singhal A, O'Connor P, Cureton A. Ergogenic effects of low doses of caffeine on cycling performanceInt J Sport Nutr Exerc Metab 2008;18:328-42.

25. Yeo S, Jentjens R, Wallis G, Jeukendrup A. Caffeine increases exogenous carbohydrate oxidation during exercise. J Appl Physiol 2005; 99: 844-50.

26. Acheson KJ, Gremaud G, Meirim I, et al. Metabolic effects of caffeine in humans: lipid oxidation or futile cycling? Am J Clin Nutr 2004;79:40-6. 
27. Heinonen, O. J., Takala, J., \& Kvist, M. Effect of carnitine loading on long-chain fatty acid oxidation, maximal exercise capacity, and nitrogen balance. Eur J Appl Physiol 1992; 65:13-17.

28. Barnett C, Costill DL, Vukovich MD, Cole KJ, Goodpaster BH, Trappe SW, Fink WJ. "Effect of L-carnitine supplementation on muscle and blood carnitine content and lactate accumulation during high-intensity sprint cycling". Int J Sport Nutr 1994; 4(3):280-8.

29. Brass, E. Supplemental Carnitina and exercise. Am J Clin Nutr 2000. 72(2): 618-23.

30. Domingues, S.F., \& Marins, J. Ergogenic aids and nutritional supplement use among resistance training practitioner in Belo Horizonte. Fit Perf J 2007, 6 (4): 218-26.

31. Antonio, J., Sanders, M.S., Kalman, D., Woodgate, D., \& Strecet, C. The effects of high-dose glutamine ingestion on weightlifting performance. J Strength Cond Res 2002;16:157-60.

32. Bakker, A., \& Berg, H. Effect of taurine on sarcoplasmic reticulum function and force in skinned fast-twitch skeletal muscle fibres of the rat. J Physiol 2002; 538(1):185-94.

33. Bemben, M., \& Lamont, H. Creatine Supplementation and Exercise Performance: Recent Findings. Sports Med 2005; 35(2), 107-25.

34. Brass, E. Supplemental L-Carnitina and exercise. Am J Clin Nutr 2000: 72(2); 618-23.

35. Burke, L., Kiens, B., \& Ivy, J. Carbohydrates and fat for training and recovery, J. Sports Sci 2004 22:15-30.

36. Candow, G., Chilibeck, P., Burke, D., Davison, S., \& Smith-Palmer, T. Effect of glutamine supplementation combined with resistance training in young adults. Eur J Appl Physiol 2001: 86: 142 - 9.

37. Collier, S. R., Collins, E., \& Kanaley, J.A. Oral arginine attenuates the growth hormone response to resistance exercise. J Appl Physiol 2006; 101: 848-52.

38. Erdman, K.A, Fung, T. \& Reimer, R.A. Influence of performance level on dietary supplementation in elite Canadian athletes. Med Sci Sports Exerc 2006; 38(2): 349-56. 